\title{
Temperature And Emissivity Separation Methods Based On TASI Data
}

\author{
Hongcheng Liu, Chuan Zhang, Fawang Ye, Jielin Zhang \\ National Key Laboratory of Remote Sensing Information and Imagery Analysis, \\ Beijing Research Institute of Uranium Geology Beijing,China \\ liuhckangl@gmail.com
}

\begin{abstract}
TASI can provide abundant useful information for the retrieval of emissivity spectrum and temperature. This paper sums up the progress of land surface temperature; summarize several methods for temperature and emissivity separation analyse the advantage and disadvantage of each method. In addition, This paper establishes regression between MMD and Bmin using 274 laboratory reflectance and field emissivity spectra, analyzes its accuracy using the data field measuring, and evaluates urban surface diurnal temperature range. Due to the effects of scale and atmosphere, the predicted value does not equal to the laboratory measurement, but the pattern of predicts is similar to the laboratory measurement; Therefore, the accuracy of this method can satisfy operational application and it is feasible to retrieve the emissivity spectrum and temperature for TASI data. Based on this relation, the TASI thermal infrared data were used for inversion and retrieval of the $\mathrm{SiO}_{2}$ content in the Xuemisitan area. The result of inversion conforms to the geological fact.
\end{abstract}

Keywords- temperature; emissivity; separation; MMD;

\section{INTRODUCTION}

Although imaging spectral technique is a powerful tool in collection, analysis, and modeling of environmental data, the application and study on thermal infrared remote sensing are not well developed. The emissivity and temperature retrieved from thermal infrared data will have an extremely important significance for scientific research and operational application. Surface emissivity is an important parameter, and emissivity spectra are often used to distinguish objective and interpret features. Land surface temperature is an important parameter for understanding land surface processes. Through measurement of surface temperatures as related to specific landscape and biophysical components and then through relating surface temperatures with energy fluxes for specific landscape phenomena or processes, (sobrino, et al., 2006). Therefore, temperature and emissivity separation are the key variables of infrared remote sensing. However, because the algorithms of temperature and emissivity separation from thermal infrared data involves solving $\mathrm{N}+1$ parameters with $\mathrm{N}$ equations, one has to assume or educe a new empirical formulation so as to make the equation complete. Generally, most algorithms vary with different assumption of emissivity. Presently, many methods are essentially successfully developed for multi-spectral thermal sensors, including the Normalized emissivity method (NEM) (Gillespie, 1985), Alpha-derived emissivity (ADE) (Kealy \& Gabell, 1990), the maximum and minimum difference method (Matsunaga, 1992), iterative spectral smooth temperature and emissivity separation (ISSTES) (Borel, 1998), and TES method (Gillespie, et al., 1998). Among those methods, TES method is composed by three modules: NEM, RAT, and MMD. It is proved that the emissivity error is within \pm 0.015 using the Aster simulated data (Yang, et al., 2010). The TES method was used to the thermal infrared multispectral data in HAPEXSahel with an error of about 3K. (Schmugge, et al. 1998).

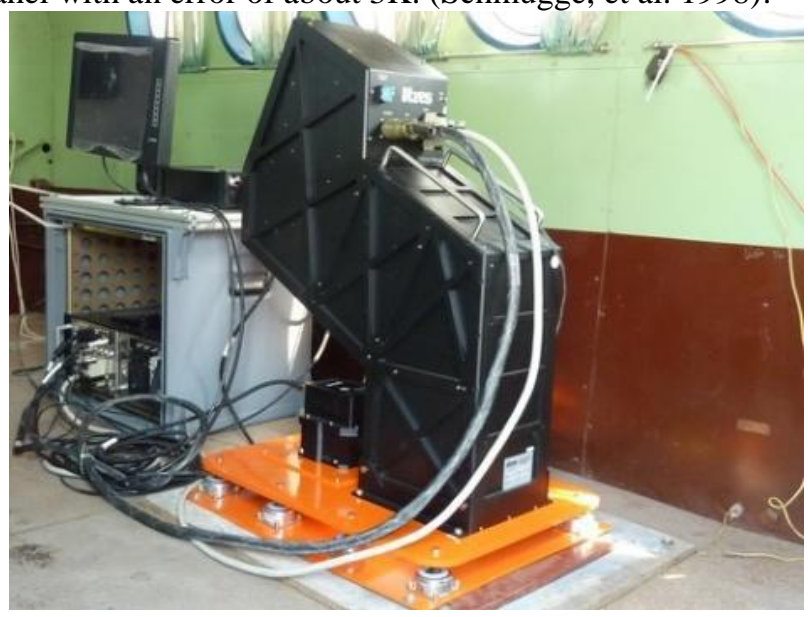

Figure 1. Appearance of TASI

However, previous studies mainly focus on the single and multispectral thermal infrared data. With the development of sensor hardware technology, hyperspectral thermal infrared data has created favorable conditions for studying on land surface emissivity and temperature. High resolution spectra includes fine surface emissivity characteristics, and aids to form more steady constraint conditions and improves the retrieval accuracy. Thermal Airborne Hyperspectral Imager (hereinafter referred to as the TASI) is one of the most advanced airborne thermal infrared imagers, which has 32 bands in the thermal infrared region $(8-11.5 \mu \mathrm{m})$ of the electromagnetic spectrum, with wavelength spacing of $0.1095 \mu \mathrm{m}, \mathrm{FWHM}$ (Full width at half maximum) of 0.0548 $\mu \mathrm{m}$, and total fields of view is $40^{\circ}$. Fig. 1 is the appearance of TASI. The radiance getting from thermal infrared sensor are the function of temperature and emissivity, so the temperature and emissivity separation is a key problem to thermal infrared remote sensing for retrieving land surface temperature. This work is to build a new empirical module based on the TASI band setting, use the TES algorithm to TASI data, and validate the reliability of the algorithm. On that basis, we further study and analyze the characteristics of surface temperature and 
emissivity, and then discuss the probability of identification using emissivity spectra.

TABLE I. TTECHNICAL INDEX OF TASI

\begin{tabular}{|c|c|}
\hline Wavelength range & $8.0-11.5 \mu \mathrm{m}$ \\
\hline Number of spectral pixels (bands) & 32 \\
\hline Number of spatial pixels & 600 \\
\hline Spectral resolution & 0.125 \\
\hline Instrument temperature & $0.3 \mathrm{k}$ \\
\hline IFOV & $40^{\circ}$ \\
\hline Instant field angle & $0.068^{\circ}$ \\
\hline SNR & 4600 \\
\hline Quantization level & $14 \mathrm{bit}$ \\
\hline Maximum power demand & $28 \mathrm{v}, 25 \mathrm{~A}$ \\
\hline Storage medium & $128 \mathrm{~GB}$ SATA hardware \\
\hline Absolute precision & $\pm 10 \%$ \\
\hline
\end{tabular}

II. THE SUMMARY OF THERMAL AIRBORNE HYPERSPECTRAL IMAGER ACQUIRED

The experiment data is important to validate and test the algorithms developed for temperature and emissivity separation using the thermal airborne hyperspectral imager. The aircraft images and the in situ data were acquired simultaneously in the framework of field campaigns at xuemisitan, Xinjiang province in 2011. The fly region lies in $46.25^{\circ} \mathrm{N}-46.41^{\circ} \mathrm{N}, 84.48^{\circ} \mathrm{E}-86.41^{\circ} \mathrm{E}$, with $30 \mathrm{~km}$ in north-south, and $150 \mathrm{~km}$ in east-west, covering an area of 150 $\mathrm{km}^{2}$. The aircraft campaign was conducted two time phase (morning, evening), two heights $(0.5 \mathrm{~km}$ and $1 \mathrm{~km})$ images of TASI were acquired. The particular flight scheme is listed in Table 1. Vertical earth observation reduces angle effect for each image. Fig. 2 is the airline chart of study area.

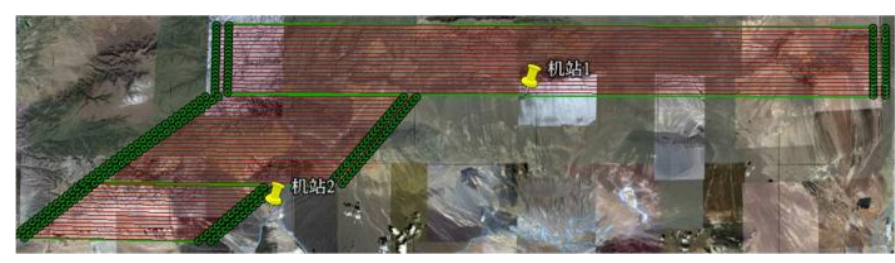

Figure 2. Perfect two canmera mosaic model

\section{THE SUMMARY OF DATA PRE-PROCESSING}

\section{A. Radiometric Calibration}

To acquire the objective radiance, radiance calibration of every band is essential before measuring the objective radiance, indicating that the digital number values (DN) recorded by the thermal infrared imaging spectrometer must be translated spectral radiance values. The radiance calibration method of TASI data is based on the assumption of a linear sensor response function, and is implemented by the means of measuring two known temperature and emissivity of standard blackbody. Firstly, it is assumed that the digital number values the TASI recorded have linear relationship with the radiance, and then radiance calibration band by band can be calculated as:

$$
L=C_{1} * D N+C_{2}
$$

In which $C_{1}$ and $C_{2}$ are gain and offset of instrument spectral response function. This procedure can be implemented automatically by the TASI data pre-procession software.

\section{B. Atmospheric correction}

TABLE II. ATMOSPHERIC CORRECTION RESULT

\begin{tabular}{|c|c|c|c|}
\hline Bandwidth & $\begin{array}{c}\text { down-welling } \\
\text { sky radiance }\end{array}$ & $\begin{array}{c}\text { up-welling } \\
\text { sky radiance }\end{array}$ & $\begin{array}{c}\text { atmospheric } \\
\text { transmittance }\end{array}$ \\
\hline 8054.799805 & 1.570391 & 1.436996 & 0.704639 \\
\hline 8164.299805 & 1.279633 & 1.343972 & 0.728063 \\
\hline 8273.799805 & 0.960569 & 0.883501 & 0.825509 \\
\hline 8383.299805 & 0.827645 & 0.560351 & 0.89338 \\
\hline 8492.799805 & 0.997773 & 0.852233 & 0.83931 \\
\hline 8602.299805 & 0.824606 & 0.549803 & 0.900881 \\
\hline 8711.799805 & 0.902223 & 0.613255 & 0.891354 \\
\hline 8821.299805 & 0.938176 & 0.775484 & 0.862111 \\
\hline 8930.799805 & 0.806228 & 0.570868 & 0.901806 \\
\hline 9040.299805 & 0.868228 & 0.572762 & 0.90248 \\
\hline 9149.799805 & 0.728385 & 0.396196 & 0.93481 \\
\hline 9259.299805 & 0.86909 & 0.388645 & 0.936446 \\
\hline 9368.799805 & 1.438639 & 0.610274 & 0.898394 \\
\hline 9478.299805 & 2.554101 & 0.702558 & 0.883154 \\
\hline 9587.799805 & 1.859053 & 0.45139 & 0.927118 \\
\hline 9697.299805 & 2.302535 & 0.57149 & 0.907187 \\
\hline 9806.799805 & 2.144888 & 0.490587 & 0.921205 \\
\hline 9916.299805 & 1.646611 & 0.406661 & 0.935818 \\
\hline 10025.79981 & 1.022588 & 0.304875 & 0.953047 \\
\hline 10135.29981 & 0.64523 & 0.283043 & 0.95674 \\
\hline 10244.79981 & 0.915736 & 0.475817 & 0.924884 \\
\hline 10354.29981 & 0.646795 & 0.346251 & 0.94633 \\
\hline 10463.79981 & 0.737505 & 0.404343 & 0.936812 \\
\hline 10573.29981 & 0.817933 & 0.467586 & 0.926361 \\
\hline 10682.79981 & 0.594021 & 0.328383 & 0.949221 \\
\hline 10792.29981 & 0.746586 & 0.42343 & 0.933504 \\
\hline 10901.79981 & 0.649995 & 0.362218 & 0.94351 \\
\hline 11011.29981 & 0.919632 & 0.578647 & 0.90733 \\
\hline 11120.79981 & 0.583569 & 0.327939 & 0.948871 \\
\hline 11230.29981 & 0.822389 & 0.463979 & 0.926207 \\
\hline 11339.79981 & 0.855365 & 0.483796 & 0.922622 \\
\hline 11449.29981 & 0.732946 & 0.42913 & 0.931326 \\
\hline Atmosph & $c 017+c i 0 n$ & \\
\hline
\end{tabular}

Atmospheric correction transforms radiance acquired by sensors into ground-leaving radiance. Assuming the land surface is Lambertian, and combining with Kirchhoff law, 
surface radiance acquired by TASI sensor can be expressed by the following formulation and the result be shown in tab2.

$$
\begin{aligned}
& L_{t o a}(\lambda)=\tau(\lambda) L_{g r d}(\lambda)+L_{a t m \uparrow}(\lambda) \\
& =\tau(\lambda)\left(\varepsilon(\lambda) B(\lambda, T)+(1-\varepsilon(\lambda)) L_{a t m \downarrow}(\lambda)\right. \\
& +L_{a t m \uparrow}(\lambda)
\end{aligned}
$$

in which $\tau(\lambda)$ is the atmospheric transmittance of channel, $L_{a t m \uparrow}(\lambda)$ is the up-welling path radiance; $L_{a t m \downarrow}(\lambda)$ is the down-welling sky radiance. $B(\lambda, T)$ is the Plank radiance at surface temperature; According to the studied region, the upwelling path radiance, the down-welling sky radiance, and the atmospheric transmittance consisting with the TASI were obtained by the MODTRAN software.

On the premise that the optimal objective temperature could be estimated, the objective emissivity can be calculated by the following equation:

$$
\varepsilon(\lambda)=\frac{L_{t o a}(\lambda)-L_{a t m \downarrow}(\lambda)}{B(\lambda, T)-L_{a t m \downarrow}(\lambda)}
$$

\section{THE ALGORITHM OF TEMPERATURE AND EMISSIVITY SEPARATION}

\section{A. Normalized Emissivity Method}

Aster team developed a new algorithm of temperature and emissivity separation (Gillespie, et al., 1998). The TES method is composed by three basic modules: NEM, Ratio, MMD.

The NEM method firstly the emissivity of each channel is assumed to be 0.99 , in order to calculate a temperature and the other emissivities. These emissivities permit iterative correction for removing the effect of down-welling sky irradiance and then an initial surface temperature can be estimated.

$$
\begin{gathered}
T_{i}=\frac{c_{2}}{\lambda_{i} \lg \left(\frac{c_{1} \varepsilon_{\max }}{\lambda_{i}^{5} \pi R_{i}}+1\right)} \\
T_{N E M}=\max \left(T_{i}\right)
\end{gathered}
$$

Re- estimate $R_{i}=L_{s}-\left(1-\varepsilon_{\max }\right) L_{a t m \downarrow}(\lambda)$ after acquire new ratio, until the temperature differs a value smaller than the pre-set threshold.

\section{B. Ratio module}

The relative spectral would be calculated by the equate(6) An important advantage of the method is that the emissivity spectral shape would be kept during the process of iteration.

$$
\beta_{i}=\frac{\varepsilon_{i}}{\varepsilon}
$$

In which, $\varepsilon_{i}$ is surface emissivity of channel $\mathrm{i} ; \bar{\varepsilon}$ is the average emissivity; $\mathrm{N}$ is the number of bands, for TASI data, $\mathrm{N}=32$.

\section{MMD module}

The empirical relationship between $\varepsilon_{\min }$ and MMD, where $\mathrm{MMD}=\max (\beta)-\min (\beta)$ is a key feature of the TES algorithm. The $\varepsilon_{\min }$ is calculated by the empirical relationship (Eq. (7)).

$$
\varepsilon_{\min }=a-b \times M M D^{c}
$$

where $\mathrm{a}, \mathrm{b}$, and $\mathrm{c}$ is the coefficients which depend on the analysis of laboratory emissivity spectra, and vary with different sensors. In order to raising the accuracy, it is essential to build a new empirical relationship based on the TASI band setting. In which, a, b, c was assigned to 1.001, $0.7799,0.8518$.

\section{RESULTS AND ANALYSIS}

\section{A. TES Result}

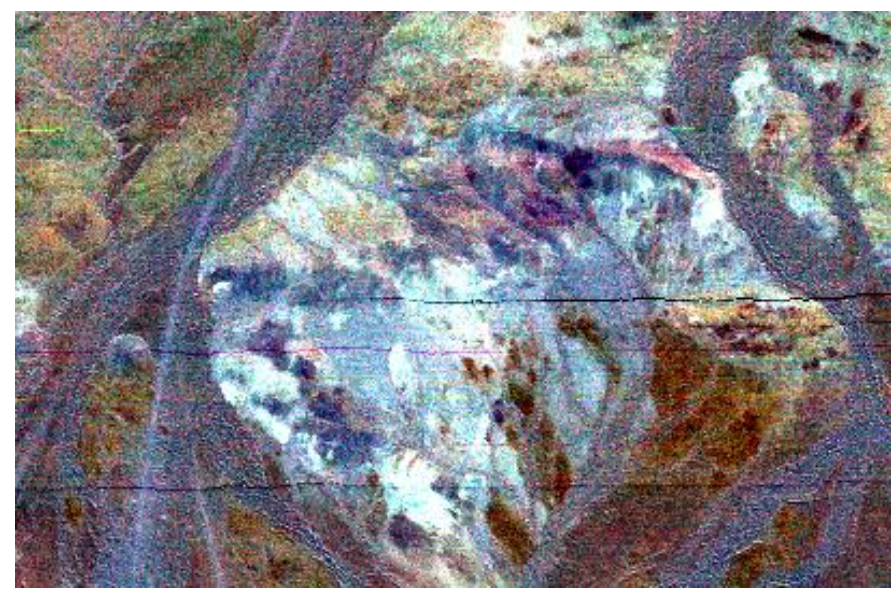

Figure 3. Emission Image

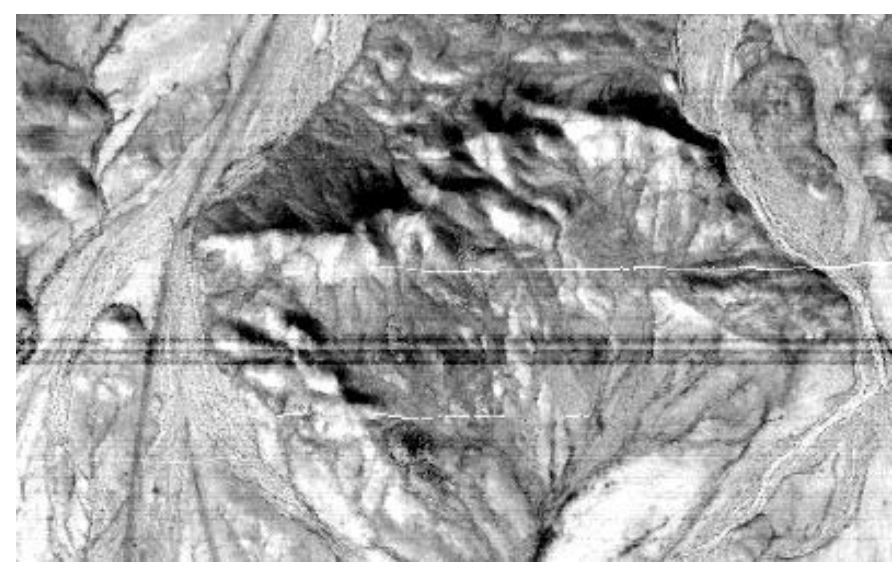

Figure 4. Temperature Image

There are two methods of data resources to evaluate the advantage and disadvantage of algorithms: using both simulated and field data. In this paper, the field data was used to analyze the retrieval accuracy from the two sides of temperature and emissivity. Fig. 3 and Fig. 4 is the temperature image and emissivity image retrieved by this method from TASI image. 


\section{B. Retrieval of The $\mathrm{SiO}_{2}$ Content}

There is a good correspondence between the thermal infrared emissivity spectral features of silicate minerals and their $\mathrm{SiO} 2$ content. The quantitative relation of the $\mathrm{SiO} 2$ content and the $\mathrm{SiO} 2$ index $(\varepsilon 12 / \varepsilon$ 13) indicating the $\mathrm{SiO} 2$ content. Basic-ultrabasic intrusions can be delinlcated using the inversion result. So this method has great application value in looking for copper-nickel deposits associated with basic-ultrabasic intrusions.

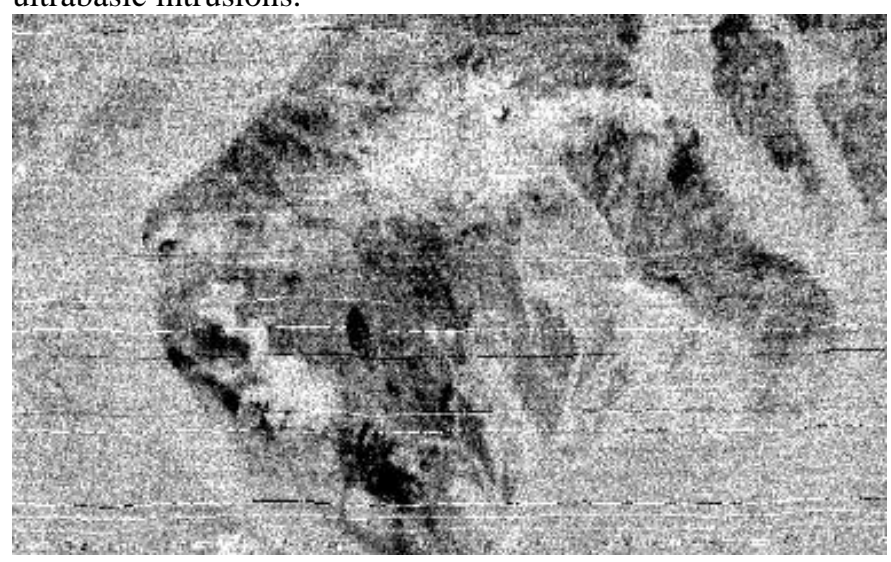

Figure 5. The Inversion Result of $\mathrm{SiO} 2$ content

\section{Fieldwork Testing}

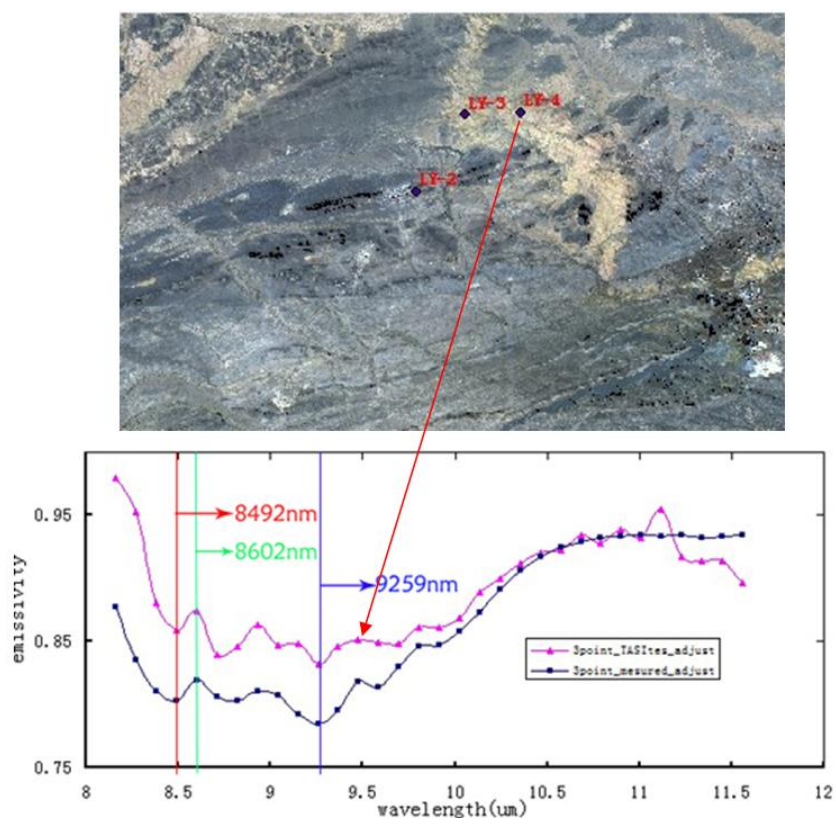

Figure 6. The Result Contrast to Field-measured Emissivity Spectral

As illustrated in Fig. 6, the retrieved emissivity value is different from the laboratory measured emissivity value, but for the similar surface type, the shapes between the two are similar. The main reason is that the scale of laboratory sample is different from the pixel scale, and the emissivities at the two scales have greater difference. It is important to effectively remove the atmospheric effect for TASI data, and meanwhile considering the sensitivity to surface roughness for laboratory measurement.

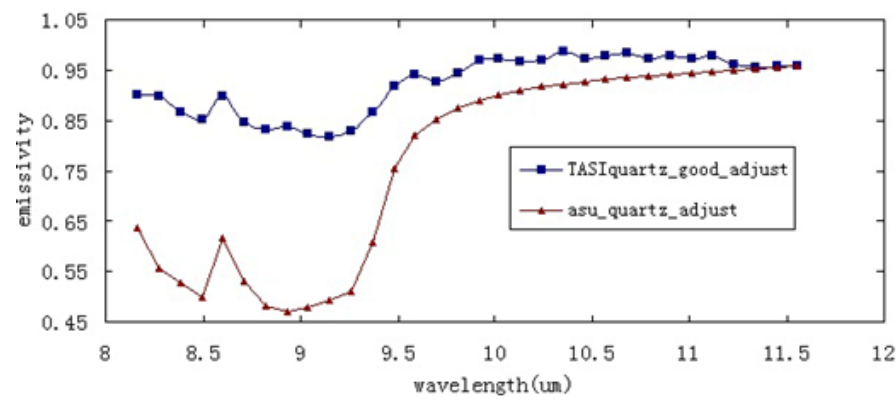

Figure 7. The Result Contrast to ASU Spectrum Stock Spectrum

\section{CONCLUSION}

To apply the algorithm of temperature and emissivity separation to thermal infrared airborne hyperspectral images, the new relationship between $\varepsilon$ min and MMD was rebuilt. The research results show that for TASI data, there was very significant exponential relationship between $\varepsilon$ min and MMD, and Eq. (7) shows that the relationship is close to a linear relation. The comparison between the retrieved emissivity of main building material and the laboratory measured emissivity demonstrates that the emissivity shapes acquired by the two methods are very similar. Considering the difference of atmosphere condition and field angle between laboratory and airborne experiment, the emissivity values of same surface type and wavelength setting are not same. The computation results of TASI broadband emissivity were in good agreement with field data and the absolute difference which shows that the reasonable accuracy.

\section{References}

[1] Zhang RH. 2009. Models and fi eld experiments for quantitative thermal infrared remote sensing. Beijing: Science Press.pp97 100

[2] Borel C C. 1998. Surface emissivity and temperature retrieval for a hyperspectral sensor. Proceedings of the International Geoscience and Remote Sensing Symposium, 1: 546 - 549

[3] Guoliang Tian, 2005. Thermal Remote Sensing. PP.171-175.

[4] Zhang Chuan, Ye Fawang. Annual Report of Beijing Research Inatitute of Uranium Geology:Study of Alteration Minal Mapping Based on the Airborne Hyperspectral Technology, Vol 28, pp.104-110.

[5] Dong Yanqing. 2012. IDL Chengxvsheji-Shuju Keshihua yu ENVI Erci Kaifa

[6] Watson K. Spectral ratio method for measuring emissivity. Remote Sens Environ, 1992, 42: 113-116

[7] Hook S J, Gabell A R, Green A A, et al. A comparison of techniques for extracting emissivity information from thermal infrared data for geologic studies. Remote Sens Environ, 1992, 42: $123-135$

[8] Becker F, Li Z L. Temperature-independent spectral indices in thermal infrared bands. Remote Sens Environ, 1990, 32: 17-33 